\title{
Minimum Cost Design of a Rectangular Box Column Composed from Cellular Plates with Welded T-Stiffeners
}

\author{
Károly Jármai and József Farkas \\ University of Miskolc, Hungary \\ \{altjar,altfar\}@uni-miskolc.hu
}

\begin{abstract}
A cantilever column is loaded by a compression force and a bending moment caused by a horizontal force. It can be derived that, in the case of uniaxial bending, the rectangular cross section is more economic than the square one. In the given numerical case, the plate thicknesses should be too large for fabrication. Therefore stiffened plates should be used. Thus, the aim of the present study is to elaborate the minimum cost design of a column with rectangular cross-section and cellular plate walls. Cellular plates are constructed from two plates and longitudinal stiffeners welded between them. Previous studies have shown that welded T-stiffeners are more economic than the halved rolled Isection stiffeners, thus, welded T-stiffeners are used.

Stress and horizontal deformation constraints are formulated. In the stress constraint the face plate buckling is avoided by using effective widths. Local buckling constraint is used for the web of T-stiffeners. Variables are as follows: heights of welded T-sections, thicknesses of stiffener webs, number of stiffeners in both directions, main dimensions of the rectangular box section, thicknesses of outer and inner face plates in smaller and larger walls. The cost function is formulated according to the fabrication sequence and consists of cost of material, welding and painting. The constrained function minimization is performed by using an effective mathematical optimization method.
\end{abstract}

Keywords: structural optimization, minimum cost design, cellular plates, columns.

\section{Introduction}

Steel columns are widely used for buildings, bridges, as supports of highways etc. A column is loaded by a compression force $N_{F}$ and a bending moment caused by a horizontal force $H_{F}=0.1 N_{F}$ (Fig.1,2). It can be derived that, in the case of uniaxial bending, the rectangular cross section is more economic than the square one. In the given numerical case, the unstiffened plate thicknesses should be too large for fabrication. Therefore stiffened plates should be used.

Results obtained for square box columns have shown that the cellular plate elements are more economic than the plates stiffened on one side (Farkas and Jármai 2008). Thus, the aim of the present study is to elaborate the minimum cost design of a column with rectangular cross-section and cellular plate walls. 


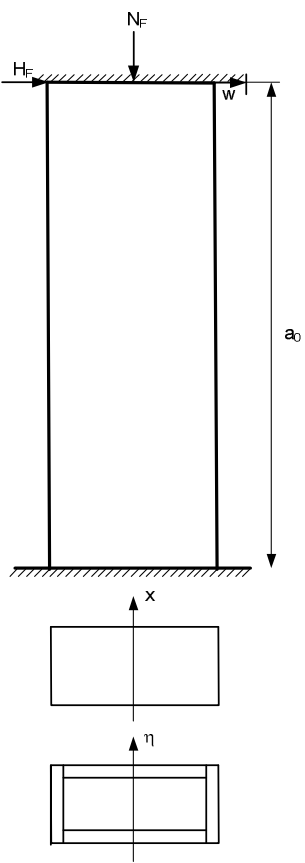

Fig. 1. Box column with walls of unstiffened and cellular plates, the two ends are built-in

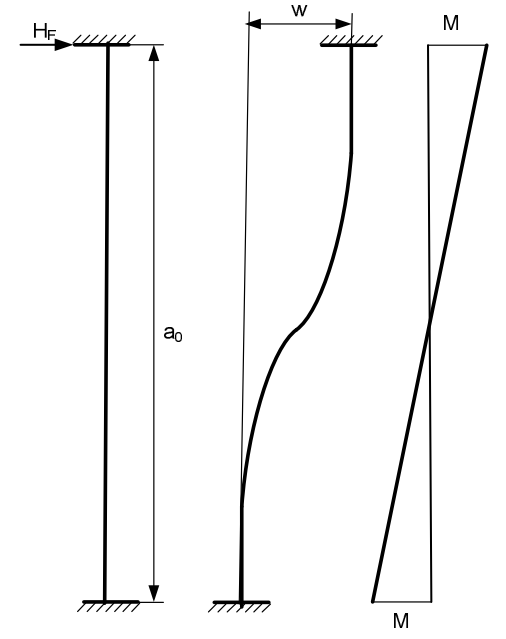

Fig. 2. Deformation and bending moment distribution of the column caused by the horizontal force

\section{Formulation of the Problem}

Numerical data: the factored compression force $N_{F}=10^{8}[\mathrm{~N}], a_{0}=15 \mathrm{~m}, f_{y}=355$ $\mathrm{MPa}, E=2.1 \times 10^{5} \mathrm{MPa}$.

Cellular plates are constructed from two plates and longitudinal stiffeners welded between them. Welded T-sections are selected for stiffeners. Figs 3 and 4 show the dimensions of cellular plate walls. Variables are as follows: height of welded Tsections $h_{1} / 2=h / 2-t_{f}, h_{11} / 2=h_{2} / 2-t_{f 1}$, thickness of stiffener webs $t_{w}$ and $t_{w 1}$, widths of stiffener flanges $b$ and $b_{1}$, number of stiffeners in both directions $n$ and $n_{1}$, main dimensions of the rectangular box section $b_{0}$ and $b_{01}$, thicknesses of outer and inner face plates in smaller and larger walls $t$ and $t_{1}$.

Ranges of variables are as follows: $t=4-40 \mathrm{~mm}, h=300-1000 \mathrm{~mm}$, $t_{f}=t, t_{f 1}=t_{1}$.

\section{Geometric Characteristics for Displacement Constraint}

Cross-sectional area for both cellular plate walls 


$$
\begin{gathered}
A=\frac{h_{1} t_{w}}{2}+b t_{f}+2 s_{y} t, s_{y}=\frac{b_{0}}{n}, h_{1}=h-2 t_{f} \\
A_{1}=\frac{h_{11} t_{w 1}}{2}+b_{1} t_{f 1}+2 s_{z} t_{1}, s_{z}=\frac{b_{01}-h-3 t}{n_{1}}, h_{11}=h_{2}-2 t_{f 1}
\end{gathered}
$$

Distance of the gravity centre

$$
z_{G}=\frac{1}{A}\left[\frac{h_{1} t_{w}}{2}\left(\frac{h_{1}+t}{2}\right)+b t_{f} \frac{h_{1}+t+t_{f}}{2}+s_{y} t\left(\frac{h_{1}}{2}+t+t_{f}\right)\right]
$$

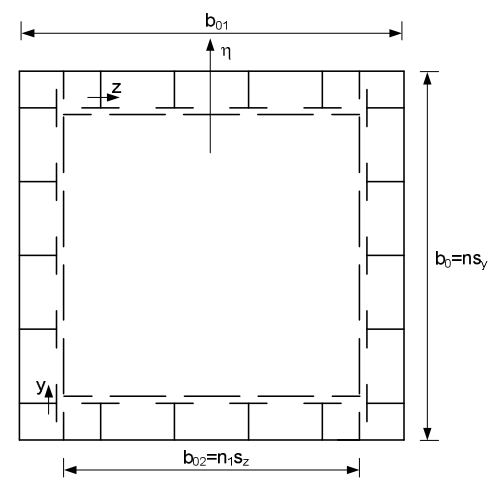

Fig. 3. Cross-section of the rectangular box column with cellular plate walls (see also Fig.1)

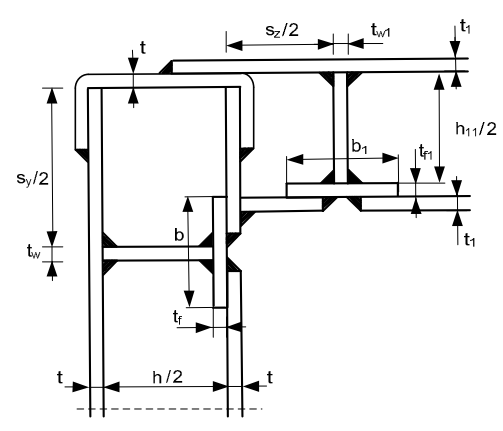

Fig. 4. Details of the corner for the box section with cellular plate walls

Moment of inertia

$$
\begin{aligned}
& I_{y}=s_{y} t z_{G}^{2}+s_{y} t\left(\frac{h_{1}}{2}+t+t_{f}-z_{G}\right)^{2}+\frac{h_{1}^{3} t_{w}}{96}+I_{y 1} \\
& I_{y 1}=\frac{h_{1} t_{w}}{2}\left(\frac{h_{1}+t}{2}-z_{G}\right)^{2}+b t_{f}\left(\frac{h_{1}+t+t_{f}}{2}-z_{G}\right)^{2}
\end{aligned}
$$

Moment of inertia of the whole rectangular box section for axis $\eta$

$$
\begin{gathered}
I_{\eta}=2 n I_{y}+2 n A\left(\frac{b_{01}}{2}-z_{G}\right)^{2}+2 \frac{b_{01}^{3} t_{1}}{12}+2 \frac{t_{1}}{12}\left(b_{01}-\frac{h_{1}}{2}-t-t_{f}\right)^{3}+2 I_{\eta 1} \\
I_{\eta 1}=2\left(\frac{h_{11} t_{w 1}}{2}+b_{1} t_{f 1}\right) s_{z}^{2} \frac{n_{1}\left(n_{1}+2\right)\left(n_{1}+1\right)}{24}
\end{gathered}
$$

The displacement constraint is given as 


$$
w=\frac{H_{F} a_{0}{ }^{3}}{12 E \gamma_{M} I_{x}} \leq \frac{a_{0}}{\phi}=15 \mathrm{~mm} \quad I_{\eta} \geq I_{0}=\frac{H_{F} L^{2} \phi}{12 E \gamma_{M}}
$$

\section{Geometric Characteristics for Stress Constraint}

The local buckling of face plates is avoided by considering effective plate widths according to Eurocode 3 (2007):

$$
\begin{gathered}
A_{e}=\frac{h_{1} t_{w}}{2}+b t_{f}+2 s_{y e} t, s_{y}=\frac{b_{0}}{n}, h_{1}=h-2 t_{f}, s_{y e}=\rho_{y} s_{y} \\
\rho_{y}=\frac{\lambda_{p y}-0.22}{\lambda_{p y}^{2}} \quad \text { if } \quad \lambda_{p y}=\frac{s_{y}}{56.8 \varepsilon t} \geq 0.673, \varepsilon=\sqrt{\frac{235}{f_{y}}} \\
\rho_{y}=1 \quad \text { if } \quad \lambda_{p y}<0.673
\end{gathered}
$$

The other formulae are similar to those in Section 3, but $s_{e}$ should be used instead of $s$.

\section{Constraint on Local Buckling of Stiffener Webs}

The webs are subject to uniform compression. According to Eurocode 3

$$
\frac{h_{1}}{2 t_{w}} \leq 42 \varepsilon_{1}, \varepsilon_{1}=\sqrt{\frac{235}{\sigma}} \text { and } \frac{h_{11}}{2 t_{w 1}} \leq 42 \varepsilon_{1}
$$

\section{Fabrication Constraints}

In order to allow the welding of stiffener web to the base plate the distance between the stiffener flanges is prescribed as $300 \mathrm{~mm}$ :

$$
n \leq \frac{b_{0}}{300+b}, n_{1} \leq \frac{b_{02}}{300+b_{1}}
$$

\section{Cost Function}

The cost function is formulated according to the fabrication sequence.

(1) Welding of outer face plates with butt welds (SAW - submerged arc welding). A plate element has sizes of 6000x1500 mm or less.

Plate of sizes $a_{0} \times b_{0}$ : volume $V_{0}=a_{0} b_{0} t$, weld length $L_{W 0}=2 b_{0}+(q-1) a_{0}$,

$$
K_{W 0}=k_{W}\left(\Theta \sqrt{3 q \rho V_{0}}+1.3 C_{W} t^{n_{0}} L_{W 0}\right) k_{W}=1.0 \$ / \mathrm{min}
$$


$q$ is the number of plate elements in the direction of $b_{0}$ so that $b_{0} / q \leq 1500 \mathrm{~mm}$.

The factor of complexity of the assembly is taken as $\Theta=2$.

$$
\begin{gathered}
\text { For } t<11 \quad C_{W}=0.1346 \times 10^{-3}, n_{0}=2 \\
\text { for } \quad t \geq 11 \quad C_{W}=0.1033 \times 10^{-3}, n_{0}=1.904 \\
\text { Plate of sizes } \quad a_{0} x b_{01}: V_{01}=a_{0} b_{01} t_{1} ; L_{W 01}=2 b_{01}+\left(q_{1}-1\right) a_{0} \\
K_{W 01}=k_{W}\left(\Theta \sqrt{3 q_{1} \rho V_{01}}+1.3 C_{W} t_{1}^{n_{0}} L_{W 01}\right)
\end{gathered}
$$

$q$ and $q_{1}$ are the numbers of plate strips of width smaller than $1500 \mathrm{~mm}$.

(2) Welding of stiffeners' webs to outer face plates and to flange with double fillet welds (GMAW-CO2 gas metal arc welding with $\mathrm{CO}_{2}$ ).

Plate of sizes $a_{0} \mathrm{x} b_{0}$ :

$$
\begin{aligned}
V_{1} & =\left(\frac{h_{1}}{2} t_{w}+b t_{f}\right) a_{0} n+V_{0}, L_{W 1}=4 a_{0} n \\
K_{W 1} & =k_{W}\left(\Theta \sqrt{(2 n+1) \rho V_{1}}+1.3 \times 0.3394 \times 10^{-3} a_{W}^{2} L_{W 1}\right)
\end{aligned}
$$

$a_{W}=0.4 t_{w}$ but $a_{W \min }=4 \mathrm{~mm}$

Plate of sizes $a_{0} \times b_{01}$ :

$$
\begin{aligned}
& V_{11}=\left(\frac{h_{11}}{2} t_{w 1}+b_{1} t_{f 1}\right) a_{0} n_{1}+V_{01}, L_{W 11}=4 a_{0} n_{1} \\
& K_{W 11}=k_{W}\left(\Theta \sqrt{\left(2 n_{1}+1\right) \rho V_{11}}+1.3 \times 0.3394 \times 10^{-3} a_{W 1}^{2} L_{W 11}\right)
\end{aligned}
$$

$a_{W 1}=0.4 t_{w 1}$ but $a_{W 1 \min }=4 \mathrm{~mm}$

(3) Welding of inner plate strips of width $s_{y}$ and $s_{z}$ from 3-3 parts with butt welds excluding the outside strips:

$$
\begin{gathered}
V_{2}=a_{0} s_{y} t \\
K_{W 2}=(n-1) k_{W}\left(\Theta \sqrt{3 \rho V_{2}}+1.3 C_{W} t^{n_{0}} 2 s_{y}\right) \\
V_{21}=a_{0} s_{z} t_{1} \\
K_{W 21}=\left(n_{1}-1\right) k_{W}\left(\Theta \sqrt{3 \rho V_{21}}+1.3 C_{W} t_{1}^{n_{0}} 2 s_{z}\right)
\end{gathered}
$$

(3a) Welding of the outside strips of width $s_{y} / 2$ and $s_{z} / 2$ :

$$
\begin{aligned}
V_{2 a} & =a_{0} s_{y} t / 2, V_{21 a}=a_{0} s_{z} t_{1} / 2 \\
K_{W 2 a} & =2 k_{W}\left(\Theta \sqrt{3 \rho V_{2 a}}+1.3 C_{W} t^{n_{0}} s_{y}\right)
\end{aligned}
$$




$$
K_{W 21 a}=2 k_{W}\left(\Theta \sqrt{3 \rho V_{21 a}}+1.3 C_{W} t_{1}^{n_{0}} s_{z}\right)
$$

(4) Welding of inner face plate strips to the stiffener flanges with double fillet welds:

$$
\begin{gathered}
V_{3}=V_{1}+a_{0} b_{0} t, L_{W 2}=2 a_{0} n \\
K_{W 3}=k_{W}\left(\Theta \sqrt{(n+2) \rho V_{3}}+1.3 \times 0.3394 \times 10^{-3} a_{W 2}^{2} L_{W 2}\right)
\end{gathered}
$$

$a_{W 2}=0.7 t$ but $a_{W 2 \min }=3 \mathrm{~mm}$

$$
\begin{gathered}
V_{31}=V_{11}+a_{0}\left(b_{01}-h-3 t\right) t_{1}, L_{W 21}=2 a_{0} n_{1} \\
K_{W 31}=k_{W}\left(\Theta \sqrt{\left(n_{1}+2\right) \rho V_{31}}+1.3 \times 0.3394 \times 10^{-3} a_{W 21}^{2} L_{W 21}\right) \\
a_{W 21}=0.7 t_{1} \quad \text { but } a_{W 21 \min }=3 \mathrm{~mm}
\end{gathered}
$$

(5) Welding of 2 U-elements to the ends of the smaller wall with 2-2 fillet welds

$$
\begin{aligned}
& A_{U}=\left(\frac{h_{1}}{2}+t_{f}+2 t+80\right) t, V_{4}=2 A_{U} a_{0}+V_{3} \\
& K_{W 4}=k_{W}\left(\Theta \sqrt{3 \rho V_{4}}+1.3 \times 0.3394 \times 10^{-3} a_{W 2}^{2} 4 a_{0}\right)
\end{aligned}
$$

(6) Welding of larger walls to the smaller ones with fillet welds

$$
\begin{gathered}
V_{5}=2 V_{4}+2 V_{31}, L_{W 3}=8 a_{0} \\
K_{W 5}=k_{W}\left(\Theta \sqrt{4 \rho V_{5}}+1.3 \times 03394 \times 10^{-3} a_{W 21}^{2} L_{W 3}\right)
\end{gathered}
$$

The material cost

$$
K_{M}=k_{M} \rho V_{5}, k_{M}=1.0 \$ / \mathrm{kg}
$$

The painting cost is calculated as

$$
K_{P}=k_{P} \Theta S_{P}, k_{P}=14.4 \times 10^{-6} \$ / \mathrm{mm}^{2}
$$

Surface to be painted

$$
S_{P}=2 a_{0}\left(2 b_{0}+2 b_{1}-\frac{h_{1}}{2}-t_{f}-2 t-\frac{h_{11}}{2}-t_{f 1}-2 t_{1}\right)
$$

The total cost

$$
\begin{aligned}
& K=K_{M}+2\left(K_{W 0}+K_{W 01}+K_{W 1}+K_{W 11}+K_{W 2}+K_{W 2 a}+K_{W 21}+K_{W 21 a}\right)+ \\
& \quad+2\left(K_{W 3}+K_{W 31}\right)+K_{W 4}+K_{W 5}+K_{P}
\end{aligned}
$$




\section{Results of the Optimization}

The optimization is made using the Particle Swarm Optimization (PSO) technique. PSO is a population based stochastic optimization technique developed by Eberhart and Kennedy (1995), inspired by social behaviour of bird flocking or fish schooling. If one of the members of the swarm sees a desirable path to go, the rest of the swarm will follow quickly. Every member of the swarm searches for the best in its locality learns from its own experience.

Each particle keeps track of its coordinates in the problem space which are associated with the best solution (fitness) it has achieved so far. (The fitness value is also stored.) This value is called pbest. Another "best" value that is tracked by the particle swarm optimizer is the best value, obtained so far by any particle in the neighbours of the particle. This location is called lbest. when a particle takes all the population as its topological neighbours, the best value is a global best and is called gbest.

The particle swarm optimization concept consists of, at each time step, changing the velocity of (accelerating) each particle toward its pbest and lbest locations. In the calculation the number of particles was 500. The detailed description of the technique is available in Farkas and Jármai (2008). The results are as follows:

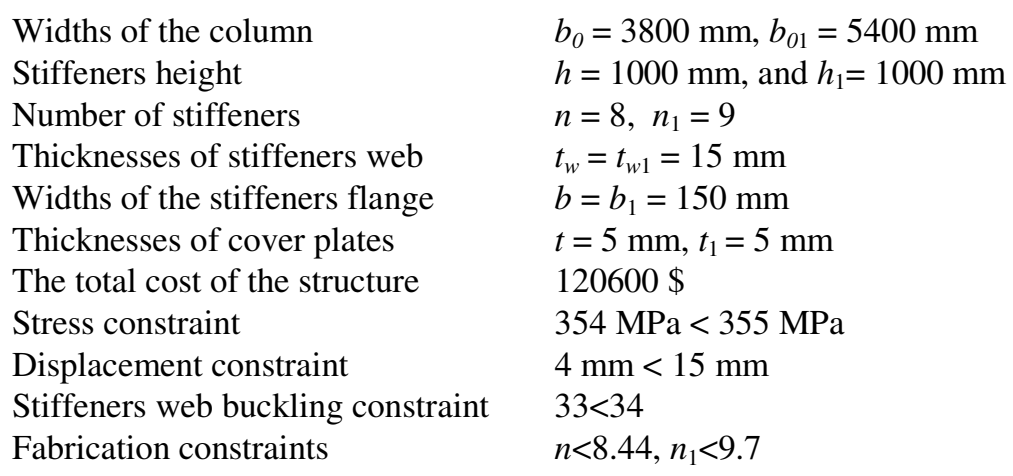

\section{Conclusions}

Cellular plates are constructed from two base plates and longitudinal stiffeners welded between them. A cantilever column is loaded by a compression force and a bending moment caused by a horizontal force. It can be derived that, in the case of uniaxial bending, the rectangular cross section is more economic than the square one. Stress and horizontal deformation constraints are formulated. In the stress constraint the face plate buckling is avoided by using effective widths. Local buckling constraint is used for the web of T-stiffeners. The cost function contains the cost of material, assembly and welding and is formulated according to the fabrication sequence. Particle Swarm Optimization is used to find the optimum. 
Acknowledgements. The research was supported by the Hungarian Scientific Research Fund OTKA T 75678 and by the TÁMOP 4.2.1.B-10/2/KONV-2010-0001 entitled "Increasing the quality of higher education through the development of research - development and innovation program at the University of Miskolc supported by the European Union, co-financed by the European Social Fund."

\section{References}

Eurocode 3, Design of steel structures. Part 1-1: General rules and rules for buildings (2009) Eurocode 3, Design of steel structures. Part 1-5: Plated structural elements (2007)

Farkas, J., Jármai, K.: Design and optimization of metal structures. Horwood Publishing, Chichester (2008)

Kennedy, J., Eberhart, R.C.: Particle swarm optimization. In: Proc. IEEE Int'l Conf. on Neural Networks, IV, pp. 1942-1948. IEEE Service Center, Piscataway (1995) 